\title{
Engineering yellow fluorescent protein probe for visualization of parallel DNA G-quadruplex
}

\author{
Tuom TT Truong ${ }^{1}$, Trang PT Phan ${ }^{1}$, Linh TT Le ${ }^{2}$, Dung H Nguyen ${ }^{3}$, Hoang D Nguyen ${ }^{1}$, Dung T Dang ${ }^{1,2, *}$
}

${ }^{1}$ Center for Bioscience and Biotechnology, University of Sciences, VNU-HCM, Viet Nam

${ }^{2}$ Faculty of Biotechnology, Ho Chi Minh City Open University, Viet Nam

${ }^{3}$ Institue of Tropical Biology, VAST, Viet Nam

\section{Correspondence}

Dung T Dang, Center for Bioscience and Biotechnology, University of Sciences, VNU-HCM, Viet Nam

Faculty of Biotechnology, Ho Chi Minh City Open University, Viet Nam

Email: dung.dthanh@ou.edu.vn

\section{History}

- Received: 11 September 2018

- Accepted: 31 October 2018

- Published: 09 November 2018

DOI :

https://doi.org/10.32508/stdj.v21i3.461

\section{Check for updates}

\section{Copyright}

(c) VNU-HCM Press. This is an openaccess article distributed under the terms of the Creative Commons Attribution 4.0 International license.

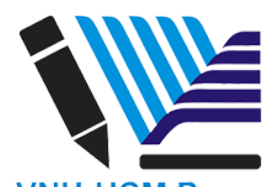

VNU-HCM Press

\begin{abstract}
Introduction: The formation of G-quadruplex plays a key role in many biological processes. Therefore, visualization of G-quadruplex is highly essential for design of G-quadruplex-targeted small molecules (drugs). Herein, we report on an engineered fluorescent protein probe which was able to distinguish G-quadruplex topologies. Methods: The fluorescent protein probe was generated by genetically incorporating yellow fluorescent protein (YFP) to RNA helicase associated with AUrich element (RHAU) peptide motif. Results: This probe could selectively bind and visualize parallel G-quadruplex structure (T95-2T) at high affinity (Kd 130 nM). Visualization of the parallel Gquadruplex by RHAU-YFP could be easily observed in vitro by using normal Gel Doc or the naked eye. Conclusion: The YFP probe could be encoded in cells to provide a powerful tool for detection of parallel G-quadruplexes both in vitro and in vivo.

Key words: DNA, RHAU, Yellow Florescent Probe, Fluorescent
\end{abstract}

\section{INTRODUCTION}

G-quadruplexes are high-order DNA or RNA formed from G-rich sequences that can fold into four singlestranded DNA or RNA structures ${ }^{1}$. G-quadruplex structures are highly polymorphic: the four strands of the G-tetrad core can be parallel (oriented in the same direction), or nonparallel with i) three in one direction and one in the other or ii) two in one direction and two in the other ${ }^{2}$ (Figure 1). Computational calculation predicts that the possible formation of G-quadruplexes in the human genome might contain over 300,000 sequences $^{2-4}$. G-quadruplexes are mostly present in telomeres of genomes which consist of 5 to $10,000 \mathrm{bp}$ of G-rich repeats (TTAGGG). In addition, G-quadruplexes are found in the promoter region of genes. G-quadruplexes have also been found in the $5^{\prime}$ untranslated region (5'-UTR) of encoded mRNAs.

In cellular systems, the formation of G-quadruplex plays a crucial role in many biological processes, such as replication, transcription, translation, and telomeric maintenance ${ }^{5,6}$. During replication, G-rich sequences have a chance to form G-quadruplexes because the DNA is transiently single-stranded which inhibits the replication process, resulting in genome instability. In human chromosomes, the 3'overhang single strand of telomeres (around 100 to $280 \mathrm{nt}$ ) are favorable to form G-quadruplexes that may inhibit telomerase activity, leading to telomere shortening $^{7-9}$. Therefore, the presence of G-quadruplexes in the human genome is considered to be a new molecular target for cancer therapeutics ${ }^{10,11}$.

Visualization of G-quadruplexes in DNA is highly essential for the design of G-quadruplex-targeted small molecules (drugs). Small molecule probes, such as bisquinolinium/thiazole orange and acetylenebridged 6,8-purine dimer, have been developed for visualization of G-quadruplexes ${ }^{12}$. These molecules can turn on their fluorescence when binding to Gquadruplexes. In recent years, visualization of Gquadruplexes by proteins have also been made possible via the use of antibodies which selectively recognize and bind to G-quadruplexes with high affinity, thus allowing the location of the G-quadruplex in the genomic telomeres to be discerned ${ }^{13}$. Specific recognition of parallel G-quadruplexes by the RNA helicase associated with AU-rich element (RHAU) protein has also been reported ${ }^{14}$. The full length of RHAU protein (1008 aa) can bind parallel G-quadruplexes and unwind G-quadruplex structure in the presence of ATP. However, only the N-region of RHAU peptide (without helicase domain) is able to selectively bind and stabilize parallel G-quadruplex structure ${ }^{14}$.

Previously, we developed the cyan fluorescent protein (CFP) probes, by fusing RHAU peptide motif to CFP, which can selectively bind and distinguish G-quadruplex topologies (parallel and non-parallel structures $)^{15}$. Nevertheless, more advanced development of fluorescent protein probes with different 

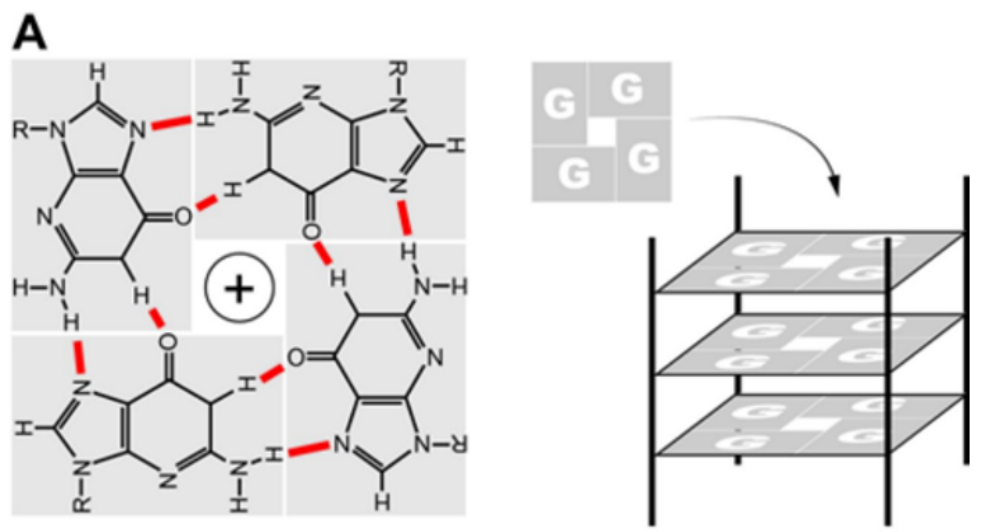

B
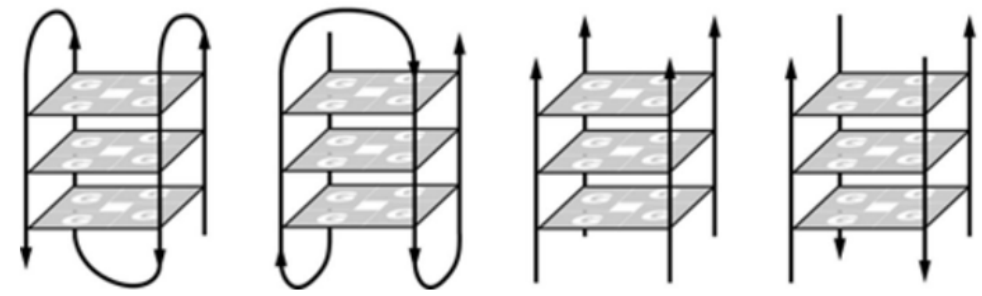

Figure 1: Schematic of G-quadruplex. A) G-quadruplex structure is formed in DNA and RNA in the presence ofcation $\mathrm{K}^{+}$or $\mathrm{Na}^{+}$. B) G-quadruplexes with differenttopologies: parallel and non-parallel ${ }^{3}$.

physical properties are still needed for optimal visualization of G-quadruplexes. In this study, we report on an engineered yellow fluorescent protein (YFP) probe (called RHAU-YFP) which incorporates YFP and RHAU (53 aa) peptide motif. This probe could selectively bind and visualize parallel G-quadruplex structure.

\section{METHODS}

\section{Construction of plasmid}

The YFP probe with RHAU peptide motif was generated by incorporating RHAU peptide and YFP. DNA encoding for YFP was amplified by PCR, using $\mathrm{pHT} 584^{16}$ containing YFP gene as the template, and primer pairs ON1 (5'-gca gat ctaggcggcggcagcatg gtg agcaagggc gag gag-3') and ON2 (5'-cag act cgagtt act tgt aca gctcgtccatgccga ga-3') (IDT, Inc., Singapore). The PCR product was cloned into treated pETduet1-RHAU ${ }^{15}$ at BglII and XhoI (New England Biolabs, Ipswich, MA, USA) sites, resulting in plasmid pETduet1-RHAU-YFP (coding for protein RHAUYFP).

\section{Protein expression and purification}

Plasmid pETduet1-RHAU-YFP (coding protein RHAU-YFP) was transformed into the host of E.coli strain BL21 (DE3). The bacteria were cultured in $\mathrm{LB}$ medium containing $200 \mu \mathrm{g}$ of ampicillin at $37^{\circ} \mathrm{C}$, $200 \mathrm{rpm}$. When reaching an OD600 of 0.6, IPTG (Sigma Aldrich, St. Louis, MO, USA) was added to a final concentration of $0.3 \mathrm{mM}$. The cells were then incubated overnight at $16^{\circ} \mathrm{C}, 250 \mathrm{rpm}$ before being harvested. The pellet was resuspended into the BugBuster protein extraction reagent (EMD Millipore, Burlington, MA, USA) plus benzonase nuclease to degrade DNA and RNA. The insoluble debris was removed by centrifugation at 20,000 $\mathrm{rpm}, 4^{\circ} \mathrm{C}$. The soluble fraction was applied to the His-tag column (ThermoFisher Scientific, Waltham, MA, USA) through gravity flow. Following that, the column was washed with 20 column volumes of 20 $\mathrm{mM}$ Tris- $\mathrm{HCl}, 100 \mathrm{mM} \mathrm{NaCl}$ and $10 \mathrm{mM}$ imidazole buffer. The column was then eluted with $20 \mathrm{mM}$ Tris- $\mathrm{HCl}, 100 \mathrm{mM} \mathrm{NaCl}$ and $200 \mathrm{mM}$ imidazole buffer. The imidazole in the buffer of the protein was removed using the Amicon Ultra-15 centrifugal filter (EMD Millipore). The homogeneous protein was collected and analyzed by SDS-PAGE. 
Gel mobility shift assay for determination of binding affinity

Gel mobility shift assay was performed using native PAGE of $10 \%$ acrylamide in $1 \mathrm{X}$ TBE (Tris-borateEDTA), $20 \mathrm{mM}$ potassium phosphate, $100 \mathrm{mM}$ potassium chloride, pH7.5. A fluorescein (FAM)-labelled parallel G-quadruplex T95-2T (50 nM) (IDT, Inc.) was incubated with increasing protein concentrations: $0,5,15,40,60,120,500$ and $1000 \mathrm{nM}$.

The gel binding data of the proteins and DNA were fitted using the following equation ${ }^{14}$ :

$\alpha=\frac{\left(K_{d}+a+b\right)-\left[\left(K_{d}+a+b\right)^{2}-4 a b\right]^{1 / 2}}{2 a}$ where a represents the DNA concentration, $\mathbf{b}-$ the protein concentration,

$\alpha$ - the fraction of bound DNA, and $K_{d}$ - the dissociation constant for DNA-protein interaction (DNA + protein $\leftrightarrow$ complex).

Visualization of parallel G-quadruplex by optical technique

Both the parallel G-quadruplex (T95-2T: 5'-TTGGGTGGGTGGGTGGGT-3')

non-parallel G-quadruplex (Htelo:

TAGGGTTAGGGTTAGGGTTAGGGTT-3')

were chemically conjugated with biotin at the 3 ' end. These molecules were attached to NeutraAvidin agarose beads (ThermoFisher Scientific). The beads (consisting of both parallel and nonparallel G-quadruplexes) were then incubated with the YFP probe in eppendorf tubes. In addition, the beads (without attached DNA) were also incubated with the YFP probe as a negative control. Visualization of parallel G-quadruplex were observed with the naked eye and with Gel Doc imaging (Alpha Innotech, San Leandro, CA, USA).

\section{RESULTS}

Construction of plasmid, protein expression and purification

DNA sequence of RHAU-YFP (coding protein RHAU-YFP) in plasmid pETduet1-RHAU-YFP was confirmed by DNA sequencing. The protein RHAU-YFP was expressed in E. coli BL21 (DE3) under IPTG regulation. RHAU-YFP consisting of His-tag at C-terminus was then purified via His column. Pure protein was evaluated by SDS-PAGE (Figure 2). The molecular weight of the protein was $36,298 \mathrm{Da}$ (shifted in between the $40 \mathrm{kDa}$ and 30 $\mathrm{kDa}$ bands of the ladder). The corrected mass of RHAU-YFP was also confirmed by matrix-assisted laser desorption/ionization (MALDI) measurement (data not shown).

\section{Gel mobility shift assay for determination of binding affinity}

We examined the binding affinity of RHAU-YFP to parallel G-quadruplex by gel mobility shift assay. FAM-labelled parallel G-quadruplex T95-2T (50 nM) was incubated with increasing RHAU-YFP concentrations: $0,5,15,40,60,120,500$ and $1000 \mathrm{nM}$. Indeed, the fluorescent protein probe (RHAU-YFP) selectively recognizes and binds parallel G-quadruplex (T95-2T). The addition of RHAU-YFP to T95-2T resulted in the formation of complex RHAU-YFP/T95$2 \mathrm{~T}$, leading to a difference in migration between T95$2 \mathrm{~T}$ alone and RHAU-YFP/T95-2T complex. The size of the RHAU-YFP/T95-2T complex was found to be larger than the T95-2T alone and, thus, the proteinbound T95-2T migrated more slowly through a native gel, causing the position of the DNA T95-2T to shift (Figure 3a). Upon addition of RHAU-YFP protein probe to the parallel G-quadruplex T95-2T, the amount of free DNA T95-2T was decreased in a dosedependent manner. The RHAU-YFP protein probe displayed a low-micromolar binding affinity to T95$2 \mathrm{~T}\left(K_{d} \sim 130 \mathrm{nM}\right)$ in $\mathrm{K}^{+}$solution (Figure $\left.3 \mathbf{b}\right)$. These results demonstrate that the effect of RHAU-YFP on the binding affinity to T95-2T was linear with the binding affinity of RHAU-CFP to T95-2 $\mathrm{T}^{15}$.

\section{Visualization of parallel G-quadruplex by optical technique}

The fluorescent protein probe (RHAUYFP) was used to visualize the parallel Gquadruplex. Both parallel G-quadruplex (T95-2T: TTGGGTGGGTGGGTGGGT) and nonparallel G-quadruplex (Htelo: TAGGGTTAGGGTTAGGGTTAGGGTT) were chemically coupled with biotin which allowed these G-quadruplex molecules to attach to Neutravidin-coated agarose beads. These beads (consisting of both parallel and nonparallel G-quadruplexes) were incubated with RHAU-YFP in eppendorf tubes (Figure 4). As a negative control, the beads without DNA were also incubated with RHAU-YFP. As expected, the beads consisting of parallel G-quadruplex displayed yellow fluorescence after washing with buffer (Figure $4 \mathbf{b}$ ). In contrast, the beads consisting of nonparallel G-quadruplex and the beads alone (negative control) displayed no color after washing with buffer. These results demonstrate that RHAU-YFP could selectively recognize and visualize the parallel G-quadruplex. Interestingly, the discrimination of G-quadruplex topologies (parallel and nonparallel) by RHAU-YFP was also easily observed by the naked eye or by normal Gel Doc imaging. 


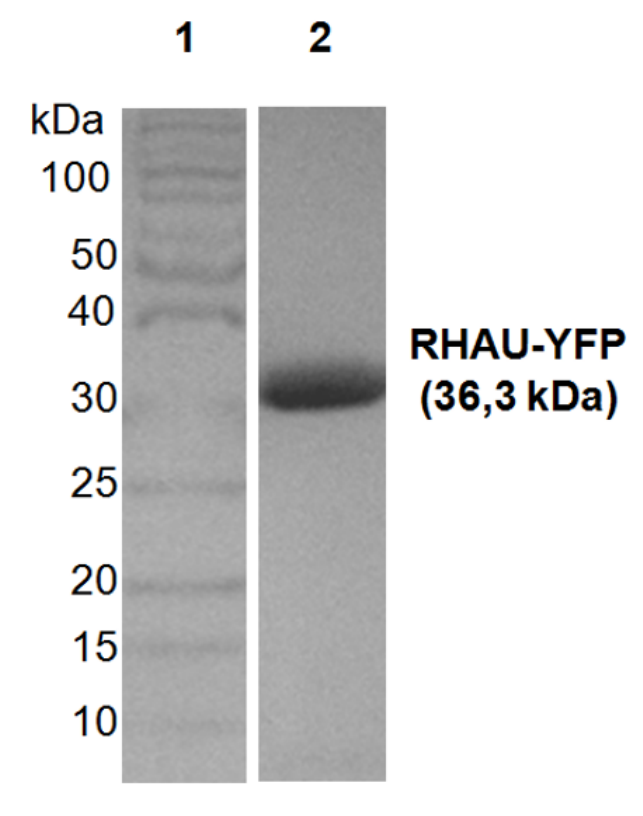

Figure 2: The purified protein (of RHAU-YFP) was confirmed by SDS-PAGE. Lane 1: $100 \mathrm{kDa}$ ladder; Lane 2: Purified RHAU-YFP.

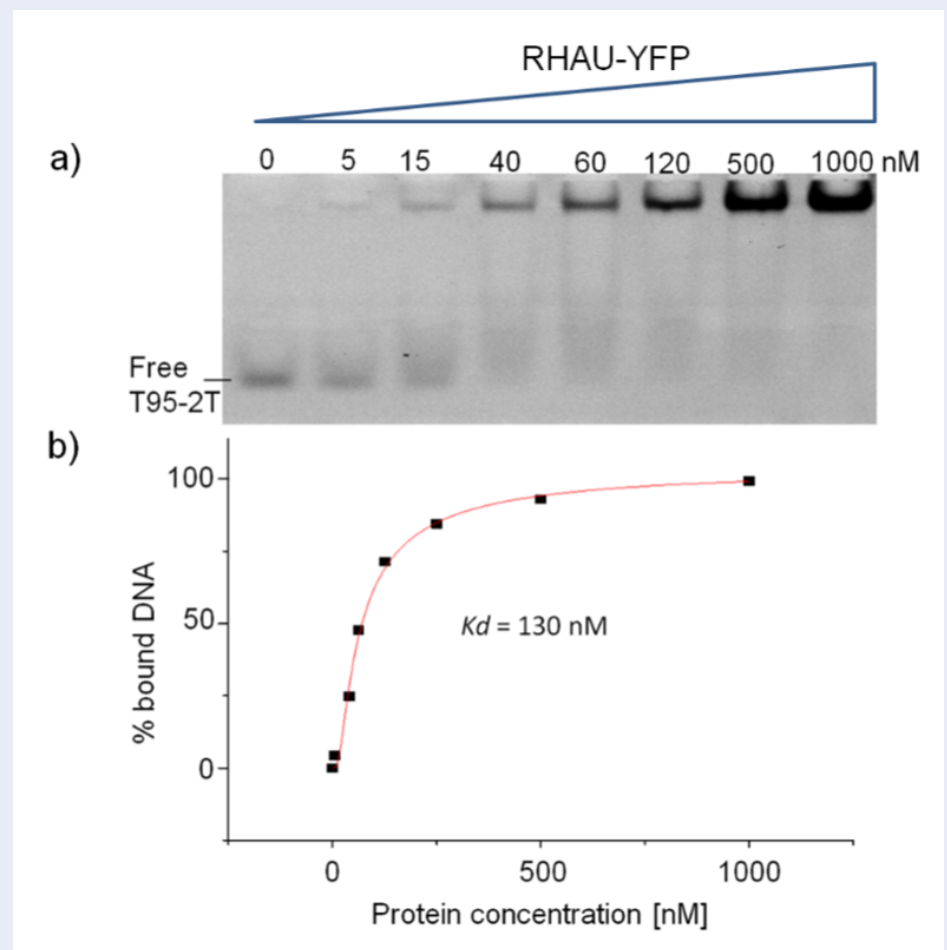

Figure 3: Gel mobility shift assay for determination of binding affinity. a) Titration of RHAU-YFP at different concentrations $(0,5,15,40,60,120,500$ and $1000 \mathrm{nM})$ to FAM-labeled T95-2T (50 nM); b) The curves were fitted by the original software following the equation above ${ }^{14}$. 
a)

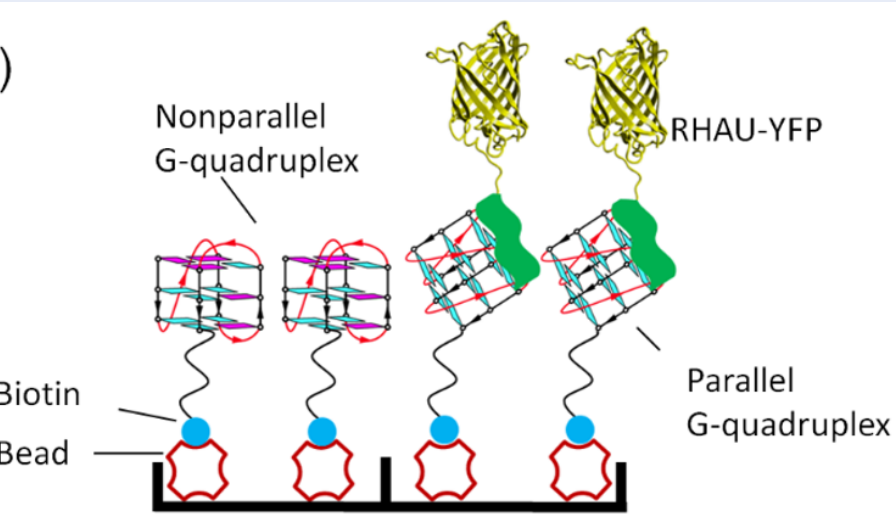

b)

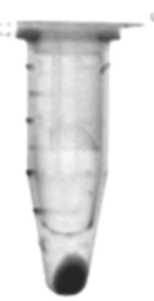

1

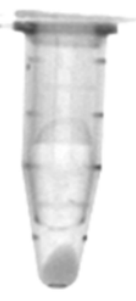

2

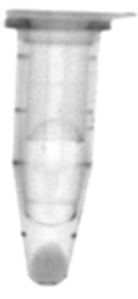

3

Figure 4: Visualization of parallel G-quadruplex by the optical technique. a) Schematic representation of selective recognition of parallel DNA G-quadruplex by RHAU-YFP. Biotin (cyan circle)-conjugated G-quadruplex attached to Neutravidin beads resulted in biotin-G-quadruplex/Neutravidin bead complexes. The fluorescent protein probe RHAU-YFP selectively binded the complex containing parallel G-quadruplex T95-2T; b) Sample 1: containing RHAU-YFP/biotin-parallel T95-2T/Neutravidin bead, sample 2: containing RHAU-YFP/biotin-nonparallel Htelo/Neutravidin bead, and sample 3: containing RHAU-YFP/Neutravidin bead (negative control). After 5 washes, all samples were visualized on a normal Gel Doc imager (Alpha Innotech), and only sample 1 displayed fluorescence.

\section{DISCUSSION}

Visualization of G-quadruplex in DNA is essential for the design of G-quadruplex-targeted small molecules. The engineered creation of a yellow fluorescent probe was performed by fusing YFP with RHAU peptide motif; this probe could selectively bind and visualize parallel G-quadruplexes. The affinity of the RHAUYFP probe to parallel G-quadruplex (T95-2T) is approximately $130 \mathrm{nM}$, which is similar to the affinity of RHAU-CFP to T95-T2 $(K d \sim 124 \mathrm{nM})^{15}$. These results of our study herein reveal that both RHAU-CFP and RHAU-YFP can be used as tools for detection of parallel G-quadruplexes at different wavelengths of emission (RHAU-CFP at emission of $475 \mathrm{~nm}$; RHAUYFP at emission of $525 \mathrm{~nm}$ ). These probes are easily manipulated and can be observed with the naked eye.

\section{CONCLUSIONS}

In conclusion, we demonstrate the generation of a yellow fluorescent protein probe by incorporating YFP to
RHAU peptide motif, resulting in RHAU-YFP. This fluorescent protein probe was shown to selectively recognize and discriminate parallel G-quadruplex and nonparallel G-quadruplex. Interestingly, visualization of parallel G-quadruplex by RHAU-YFP in vitro could be easily observed with the naked eye. Thus, the YFP probe can be genetically encoded in cells to provide a powerful tool for detection of parallel G-quadruplexes both in vitro and in vivo.

\section{COMPETING INTERESTS}

There is no conflict of interest.

\section{AUTHORS' CONTRIBUTIONS}

T.T.T.T. performed experiments under the supervision of D.T.D. All authors designed experiments, analysed data. T.T.T.T and D.T.D wrote the paper. 


\section{ACKNOWLEDGMENTS}

This research is funded by Vietnam National Foundation for Science and Technology Development (NAFOSTED) under grant number 108.02-2017.305.

\section{REFERENCES}

1. Gellert M, Lipsett MN, Davies DR. Helix formation by guanylic acid. Proceedings of the National Academy of Sciences of the United States of America. 1962;48:2013-8. Available from: DOI:10.1073/pnas.48.12.2013.

2. Burge S, Parkinson GN, Hazel P, Todd AK, Neidle S. Quadruplex DNA: sequence, topology and structure. Nucleic Acids Research. 2006;34:5402-15. Available from: DOI:10.1093/nar/ gkl655.

3. Rhodes D, Lipps HJ. G-quadruplexes and their regulatory roles in biology. Nucleic Acids Research. 2015;43:8627-37. Available from: DOI:10.1093/nar/gkv862.

4. Maizels N, Gray LT. The G4 genome. PLOS Genetics. 2013;9:e1003468. Available from: DOI:10.1371/journal.pgen. 1003468.

5. Agarwala P, Kumar S, Pandey S, Maiti S. Human telomeric RNA G-quadruplex response to point mutation in the G-quartets. The Journal of Physical Chemistry B. 2015;119:4617-27. Available from: DOI:10.1021/acs.jpcb.5b00619.

6. Agarwala P, Pandey S, Maiti S. Role of G-quadruplex located at $5^{\prime}$ end of mRNAs. Biochimica et Biophysica Acta. 2014;1840:3503-10. Available from: DOI:10.1016/j.bbagen. 2014.08.017.

7. Sun D, Thompson B, Cathers BE, Salazar M, Kerwin SM, Trent JO. Inhibition of human telomerase by a G-quadruplexinteractive compound. Journal of Medicinal Chemistry. 1997;40:2113-6. Available from: DOI:10.1021/jm970199z.

8. Read M, Harrison RJ, Romagnoli B, Tanious FA, Gowan SH, Reszka AP. Structure-based design of selective and potent $\mathrm{G}$ quadruplex-mediated telomerase inhibitors. Proceedings of the National Academy of Sciences of the United States of America. 2001;98:4844-9. Available from: DOI:10.1073/pnas. 081560598 .

9. Domenico AG, Liqian W. Dna Nanotechnology For Bioanalysis: From Hybrid Dna Nanostructures To Functional Devices; 2017.

10. Balasubramanian $\mathrm{S}$, Neidle $\mathrm{S}$. G-quadruplex nucleic acids as therapeutic targets. Current Opinion in Chemical Biology. 2009;13:345-53. Available from: DOI:10.1016/j.cbpa.2009.04. 637.

11. Buket O, Clement L, DanZhou Y. DNA G-quadruplex and its potential as anticancer drug target. Science China Chemistry. 2014;57:1605-14. Available from: DOI:10.1007/s11426-0145235-3.

12. Yang P, Cian AD, Teulade-Fichou MP, Mergny JL, Monchaud D. Engineering bisquinolinium/thiazole orange conjugates for fluorescent sensing of G-quadruplex DNA. Angewandte Chemie International Edition in English. 2009;48:2188-91. Available from: DOI:10.1002/anie.200805613.

13. Kwok CK, Balasubramanian S. Targeted Detection of G Quadruplexes in Cellular RNAs. Angewandte Chemie International Edition in English. 2015;54:6751-4. Available from: DOI:10.1002/anie.201500891.

14. Heddi $B$, Cheong VV, Martadinata H, Phan AT. Insights into G-quadruplex specific recognition by the DEAH-box helicase RHAU: solution structure of a peptide-quadruplex complex. Proceedings of the National Academy of Sciences of the United States of America. 2015;112:9608-13. Available from: DOI:10.1073/pnas.1422605112.

15. Dang DT, Phan AT. Development of Fluorescent Protein Probes Specific for Parallel DNA and RNA G-Quadruplexes. ChemBioChem. 2016;17:42-5. Available from: DOI:10.1002/ cbic.201500503.

16. Nguyen HD, Dang DT, van Dongen JL, Brunsveld L. Protein Dimerization Induced by Supramolecular Interactions with Cucurbit[8]uril. Angewandte Chemie International Edition in English. 2010;49:895-8. Available from: DOI:10.1002/anie. 200904413. 